\title{
Exploring digenic inheritance in arrhythmogenic cardiomyopathy
}

Eva König ${ }^{1}$, Claudia Béu Volpato ${ }^{1}$, Benedetta Maria Motta ${ }^{1}$, Hagen Blankenburg${ }^{1}$, Anne Picard ${ }^{1}$, Peter Pramstaller ${ }^{1}$, Michela Casella², Werner Rauhe ${ }^{3}$, Giulio Pompilio ${ }^{4}$, Viviana Meraviglia', Francisco S. Domingues ${ }^{1 *}$,

Elena Sommariva ${ }^{4^{*+}}$ and Alessandra Rossini ${ }^{1{ }^{*}+}$ (D)

\begin{abstract}
Background: Arrhythmogenic cardiomyopathy (ACM) is an inherited genetic disorder, characterized by the substitution of heart muscle with fibro-fatty tissue and severe ventricular arrhythmias, often leading to heart failure and sudden cardiac death. ACM is considered a monogenic disorder, but the low penetrance of mutations identified in patients suggests the involvement of additional genetic or environmental factors.

Methods: We used whole exome sequencing to investigate digenic inheritance in two ACM families where previous diagnostic tests have revealed a PKP2 mutation in all affected and some healthy individuals. In family members with PKP2 mutations we determined all genes that harbor variants in affected but not in healthy carriers or vice versa. We computationally prioritized the most likely candidates, focusing on known ACM genes and genes related to PKP2 through protein interactions, functional relationships, or shared biological processes.

Results: We identified four candidate genes in family 1, namely DAG1, DAB2IP, CTBP2 and TCF25, and eleven candidate genes in family 2 . The most promising gene in the second family is TTN, a gene previously associated with $A C M$, in which the affected individual harbors two rare deleterious-predicted missense variants, one of which is located in the protein's only serine kinase domain.
\end{abstract}

Conclusions: In this study we report genes that might act as digenic players in ACM pathogenesis, on the basis of co-segregation with PKP2 mutations. Validation in larger cohorts is still required to prove the utility of this model.

Keywords: Digenic inheritance, Arrhythmogenic cardiomyopathy, Exome sequencing, ACM, PKP2

\section{Background}

Arrhythmogenic cardiomyopathy (ACM) is a genetic disorder in which the ventricular myocardium is progressively replaced by fibro-fatty tissue. Since this occurs predominantly in the right ventricle, the disease is also known as arrhythmogenic right ventricular cardiomyopathy (ARVC). ACM is associated with progressive heart failure and severe ventricular arrhythmias, often leading to sudden death, especially in young people and athletes [1]. About half of the affected individuals harbor

\footnotetext{
* Correspondence: francisco.domingues@eurac.edu;

elena.sommariva@cardiologicomonzino.it; alessandra.rossini@eurac.edu

${ }^{\dagger}$ Equal contributors

'Institute for Biomedicine, Eurac Research, Affiliated Institute of the University of Lübeck, Bolzano, Italy

${ }^{4}$ Vascular Biology and Regenerative Medicine Unit, Centro Cardiologico

Monzino IRCCS, Via Parea 4, 20138 Milan, Italy

Full list of author information is available at the end of the article
}

mutations in one of the five genes of the cardiac desmosome (PKP2, JUP, DSP, DSG2, DSC2), of which mutations in PKP2 are most common. Desmosomes are intercellular junctions that confer strong cell-cell adhesion and provide a mechanical connection between cardiomyocytes. Therefore, desmosomal defects can have deleterious effects on tissue integrity. In addition, desmosomal proteins play an important role in signaling and regulation of cell proliferation and differentiation [2]. In ACM, pathogenic mechanisms include suppression of Wnt signaling and activation of the Hippo pathway [3] leading to adipogenesis. Beside desmosomal genes, mutations in eight additional genes (DES, PLN, TGFB3, CTNNA3, LMNA, TMEM43, RYR2, and TTN) have been found to cause ACM [1]. Recently, two studies reported FLNC and $\mathrm{CDH} 2$ as possible novel causative genes for ACM $[4,5]$. In most patients, ACM is inherited in an autosomal 
dominant mode with reduced penetrance (not all individuals with a causal mutation develop ACM) and variable expressivity (the severity and the nature of the symptoms may vary between affected individuals, even if they have the same causal mutation).

Autosomal dominant mutations have only been identified in up to $60 \%$ of all ACM patients [1] suggesting the existence of unknown mechanisms such as higher genetic heterogeneity, modifier genes, or cross talk between genetic background and environmental factors [6]. In fact, three loci have been mapped in ACM linkage studies, for which the causal gene has not yet been identified: ARVD3 (OMIM \%602,086) at 14q12-32.3 [7], ARVD4 (OMIM \%602,087) at 2q32.1-32.3 [8], and ARVD6 (OMIM \%604,401) at 10p14-p12 [9]. In addition, the frequency of variants associated with ACM has been found to be much higher than expected given the phenotype prevalence in the general population, suggesting that a high number of these variants are not monogenic causes of ACM [10]. In fact, recent reports have suggested digenic inheritance as an alternative disease mechanism of ACM [11-14]. In digenic inheritance the presence of two variants in two different genes is required for the manifestation of a clinical phenotype; in the absence of one of these variants, the other variant might be benign. For example, $\mathrm{Xu}$ et al. screened $198 \mathrm{ACM}$ patients for variants in the desmosomal genes. Of the 38 patients in which $P K P 2$ variants were detected, additional variants in $P K P 2$ itself (compound heterozygosity) were identified in nine patients; variants in other desmosomal genes (digenic inheritance) were identified in 13 patients. Related family members harboring a variant in just one of these genes were unaffected by ACM. The authors concluded that the disease was caused by compound heterozygosity or digenic inheritance in these patients [12]. Rasmussen et al. investigated 12 families with variants in DSG2. In three of these families, additional variants were identified in the DSP gene in affected family members. Only individuals with both variants in DSG2 and DSP were affected by ACM, leading the authors to conclude that low penetrance of desomosmal variants in ACM patients may also be explained by digenic inheritance [13]. Cooper et al. proposed that digenic inheritance may occur as a result of variants in two genes encoding different subunits of the same protein (complex); two proteins that interact functionally; are a receptor-ligand pair; are a target gene and transcription factor; or compromise the same regulatory, biosynthetic, or degradative pathway [11]. Digenic inheritance is distinct from modifier genes: in digenic inheritance, both variants individually usually do not lead to disease, whereas in modifier genes one pathogenic variant is enhanced by a putatively contributing variant of unknown significance [15]. Non-genetic factors known to influence ACM penetrance are age, male sex and intense physical activity $[16,17]$.

We performed whole exome sequencing on two families, in which diagnostic tests have identified a PKP2 mutation in affected and healthy individuals. Assuming a digenic mode of inheritance, we determined all genes, where in addition to the observed PKP2 variant a second causal variant was expected to be present in either the affected individuals or the PKP2 carriers. Filtering and prioritizing these genes, we determined four candidate genes in the first, and eleven candidate genes for digenic inheritance in the second family.

\section{Methods}

\section{Subjects}

In this study two Italian families comprising eight and four individuals, respectively, were investigated. Two individuals in the first and one individuals in the second family have been diagnosed with arrhythmogenic cardiomyopathy (ACM) according to the diagnostic task force criteria [18] (Table 1). Furthermore, previous clinically certified molecular tests on known ACM related genes have identified PKP2 variants in all affected and some healthy family members (Fig. 1).

Family 1 (Fam 1 ) consists of eight individuals in three generations of which two are affected by ACM (Fam1.III.2 and Fam1.III.3). The five individuals Fam1.I.2, Fam1.II.2, Fam1.III.1, Fam1.III.2, and Fam1.III.3 carry the heterozygous one base-pair deletion NM_004572. 3(PKP2):c.2013delC, NP_004563.2(PKP2):p.Lys672ArgfsTer12, which results in a premature stop codon after a frameshift mutation.

Family 2 (Fam2) consists of two parents and their two sons, one of which (Fam2.II.1) is affected by ACM. The male patient Fam2.II.1, his brother Fam2.II.2 and their mother Fam2.I.2 carry the heterozygous nine base pair deletion NG_009000.1(PKP2):c.2569_2577 + 41del, which crosses an exon/intron border.

In this study, ACM-diagnosed individuals are referred to as affected, healthy individuals with a PKP2 mutation are called carriers, and healthy individuals without a PKP2 mutation are called healthy.

Details on the diagnostic genetic tests are given in the Additional file 1.

\section{Data generation and computational processing}

Samples were prepared following the Nextera ${ }^{\circ}$ Rapid Capture Exome Enrichment kit protocol and were sequenced on two lanes of a HiSeq 2500 in paired end mode $(2 \times 100)$. Reads were aligned with BWA [19] and variants called with GATK [20], following the best practice recommendations. Variants were annotated with information from Ensembl [21], the ExAC project [22] (allele frequency $(\mathrm{AF})$, variants with $\mathrm{AF}<0.01$ are called 


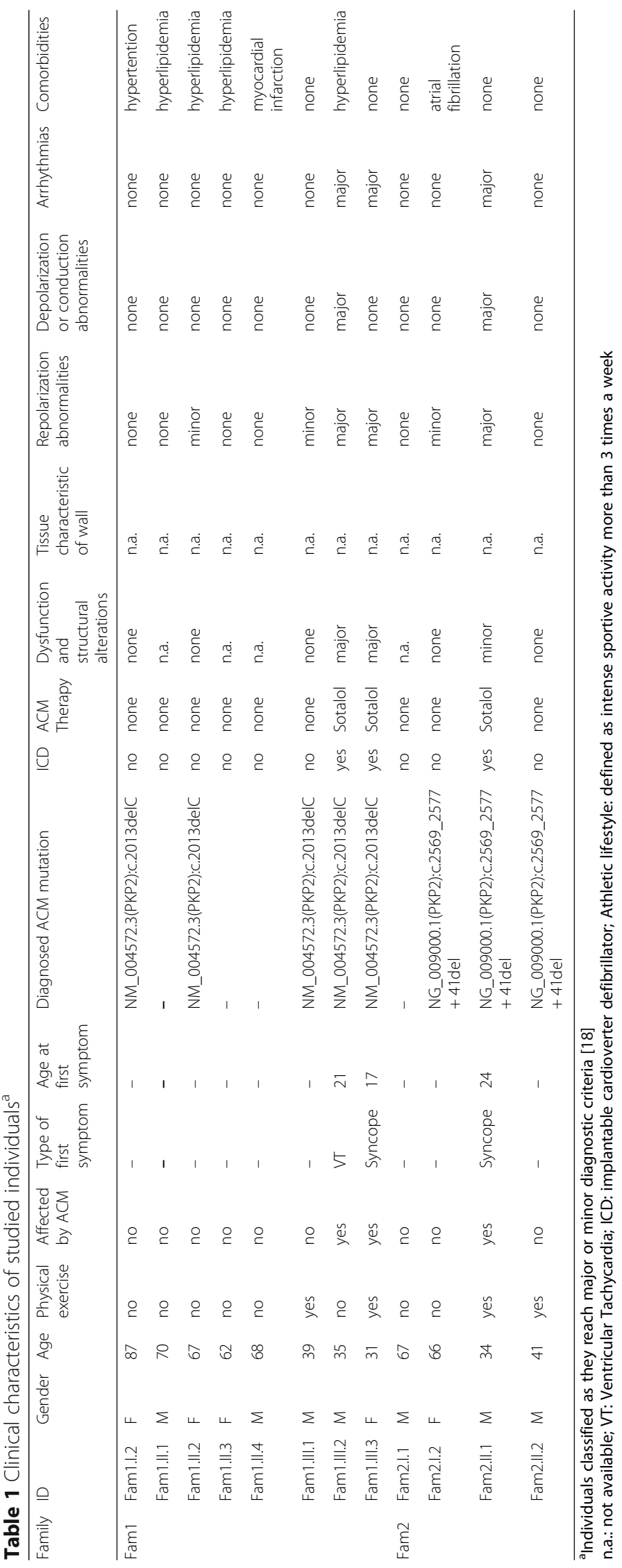




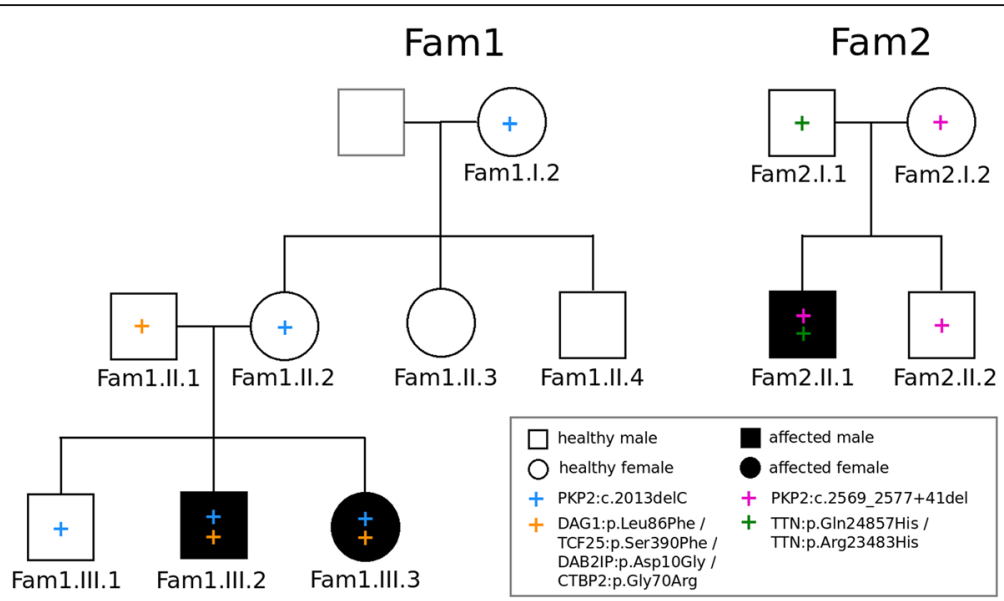

Fig. 1 Pedigrees of the two families analyzed in this study. Only labeled individuals were sequenced. +: Heterozygous variant, filled black symbols: affected individuals; white symbols with +: carrier individuals; white empty symbols: healthy individuals. Left: Family 1 (Fam 1); the blue + symbol indicates the presence of the NM_004572.3(PKP2):C.2013delC variant, the orange + symbol indicates the presence of the four Fam1 variants ENSP00000312435.2(DAG1):p.Leu86Phe, ENSP00000263347.7(TCF25):p.Ser390Phe, ENSP00000259371.2(DAB2IP):p.Asp10Gly, ENSP00000357816. 5(CTBP2):p.Gly70Arg. Right: Family 2 (Fam2); the pink + symbol indicates the presence of the NG_009000.1(PKP2):c.2569_2577+41del variant, the green + symbol indicates the presence of the ENSP00000434586.1(TTN):p.Gln24857His and the ENSP00000434586.1(TTN):p.Arg23483His variants

rare), PROVEAN deleteriousness prediction scores (variants with scores $<-2.5$ are called deleterious) [23] (for SNPs and indels), and LR.PF3 pathogenicity prediction scores [24] (for SNPs only). Copy number variations (CNVs) were called with XHMM [25]. MAESTROweb $[26,27]$ was used to predict the effect of variants on protein stability based on protein structure, where structure data were available. Sequence conservation was computed with ConSurf [28]. A detailed description is given in the Additional file 1.

\section{Family-based gene selection}

To investigate whether individuals in the two families develop ACM if they carry the PKP2 mutation and a variant that affects a second unknown gene, a set of putative causal genes was compiled in each family. All genes were determined that have a least one variant that meets the following three criteria: (i) The variant has a consequence, that is classified as either "high" (transcript ablation, splice acceptor variant, splice donor variant, stop gained, frameshift variant, stop lost, start lost, transcript amplification) or "moderate" (inframe insertion, inframe deletion, missense variant, protein altering variant) by Ensembl. (ii) The variant is either present in the family's affected individuals and not in any family's PKP2 carrier individuals or it is present in the family's $P K P 2$ carrier individuals and not in any family's affected individuals in either a dominant or a recessive mode. (iii) The variant has a coverage of at least $10 \mathrm{X}$ in all affected and carrier individuals.

In addition to the Fam 1 and Fam 2 family members, an unrelated female ACM affected individual, her carrier sister, and their carrier aunt, all carrying a heterozygous
PKP2 exon 4 deletion, were used to exclude variants as described in (ii) (see Additional file 1 for details). Variants were not filtered based on allele frequency or pathogenicity prediction. Furthermore, all genes were determined that harbored copy number variations (CNV; either a deletion or a duplication) in the affected and carrier individuals applying the same genotype selection criteria as for variants.

Each family's gene set was filtered to only include genes expressed in the heart. The RNA gene dataset was downloaded from the ProteinAtlas [29] version 15, which contains gene expression levels of 45 cell lines and 32 tissues based on RNA-seq. A gene was considered expressed in the heart, if it had an expression level of at least 5 FPKM in the heart muscle in this dataset. We call the genes/variants determined by these filtering steps Fam1 genes/variants and Fam2 genes/variants. This gene selection is visualized in Fig. 2.

\section{ACM and PKP2-related genes}

A set of 15 genes known to be involved in ACM was created by literature review $[4,5,30]$. In particular, the ACM gene set consists of the desmosomal genes PKP2, $J U P, D S P, D S G 2$, and DSC2 and the genes DES, PLN, RYR2, TGFB3, TMEM43, TTN, CTNNA3, LMNA, FLNC, and $C D H 2$.

Since PKP2 is relevant for the development of ACM in both families, we assumed that the second unknown gene is directly related to PKP2 [11]. Following the probable mechanisms of digenic inheritance described by Cooper et al. [11], a set of PKP2-related genes was compiled based on the following five criteria. (i) The two gene products form a protein complex. Gene complex 


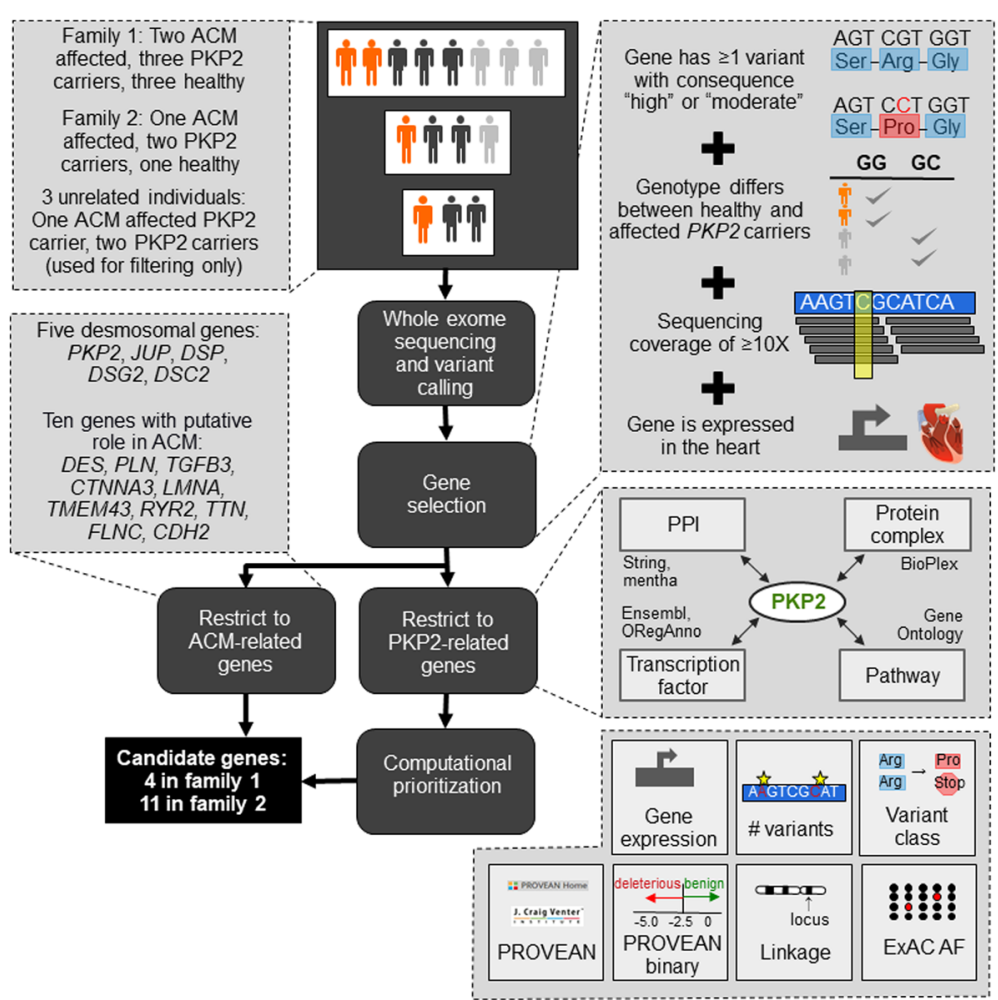

Fig. 2 Methods summary. Whole exome sequencing and variant calling was performed for each individual in both families. For each family, genes are selected that have at least one variant with a high or moderate variant impact, that differ between healthy and affected PKP2 carriers, that have a coverage of at least 10X, and that are expressed in the heart. These genes are filtered to include only genes related to ACM or PKP2 and are computationally prioritized. Results are presented in Table 2

data were downloaded from the BioPlex database [31] version 4 . Genes that interacted with $P K P 2$ with a confidence of at least 0.7 were selected. (ii) The two gene products interact functionally. PKP2 interactors were downloaded from the STRING database [32] version 10 and the mentha database [33] version 2016-08-07. STRING interactions were restricted to those with a confidence of at least 0.7. The union of PKP2 interactors from the two databases was selected. (iii) The two gene products are transcription factor and target gene. The Ensembl database [21] version 84 and ORegAnno database [34] (release 2015.12.22) were manually reviewed for transcription factors of PKP2. (iv) The two gene products participate in the same pathway. "Biological process" (BP) gene annotations from the Gene Ontology [35] (GO) were used as an approximation. All biological process annotations of PKP2 and all their annotated proteins were queried from the GO database version 2016.7 using the Dintor GOAnnotator tool [36]. Processes were restricted to those where at least half of their annotated genes were expressed in the heart and all genes annotated to these processes were selected, creating a set of genes that share a biological function with PKP2. (v) The two genes are paralogs. Though not specifically listed as a mechanism of digenic inheritance, Cooper et al. discussed that paralogous genes might provide a level of redundancy by resuming gene function in case of a disruption [11]. Therefore, genes paralogous to PKP2 were queried from Ensembl 86.

The set of genes that meet all five criteria was named the PKP2-related genes. Analogous to the Fam1 and Fam2 gene sets, PKP2-related genes were restricted to those expressed in the heart.

The intersection of Fam1 and Fam2 genes with the PKP2-related genes was prioritized using the Dintor MetaRanker tool [36], by equally weighting gene expression, number of variants, variant class (consequence class "high" was rated higher than class "moderate"), minimum ExAC allele frequency of the gene's variant(s), minimum PROVEAN score of the gene's variant(s), binary prediction (neutral or deleterious) of this PROVEAN score, and presence in a linkage region (see Fig. 2). The LR.PFS3 model [24] was not used in the ranking since it is not defined for indels.

\section{Results}

Whole exome sequencing

For each of the eight samples relevant to this study (Fam1.I.2, Fam1.II.2, Fam1.III.1, Fam1.III.2, Fam1.III.3, 
Fam2.I.2, Fam2.II.1, and Fam2.II.2), an average of 54.3 \pm 15.3 million reads was generated. The mean base quality was well above $30 \mathrm{Q}$ at all read positions, yet a drop in quality could be observed in the last 20 bases of each read. Nearly all reads (99.9\%) could be successfully mapped to the human reference genome GRCh37, resulting in a mean coverage of $24.4 \pm 3.9 \mathrm{X}$ at a mean mapping quality of $45.2 \pm 0.8 \mathrm{Q}$. On average, $85.1 \pm 3.8 \%$ of the exonic target region was covered with at least $10 \mathrm{X}$.

\section{Identification of candidate genes}

The filtering strategy described in the Methods Section and visualized in Fig. 2 resulted in 85 variants in 74 distinct genes in Fam 1 and 242 variants in 212 distinct genes in Fam2. The gene sets for both families obtained by this filtering strategy are available as Additional file 1: Table S1. No CNVs in either family met the selection criteria. Since the number of genes per family was too large to analyze in detail, we decided to restrict our analysis to known ACM related genes and then to PKP2-related genes.

From the 15 ACM-related genes, none was present in the gene set of Fam1, while TTN was in the gene set of Fam2 (Table 2). Specifically, the affected male patient Fam2.II.1 and his healthy father Fam2.I.1 harbored three distinct heterozygous missense variants in TTN. The two rare variants ENSP00000434586.1:p.Gln24857His (isoform N2B) and ENSP00000434586.1:p.Arg23483His (isoform N2B) were predicted deleterious by PROVEAN and LR.PFS3. Both variants were confirmed by Sanger sequencing (see Additional file 1). The third TTN variant ENSP00000434586.1:p.Ile3716Val (isoform N2B) is common, not conserved, and was predicted neutral by PROVEAN and LR.PFS3. TTN encodes for titin, the largest human protein, which has over 300 highly repetitive independently folding domains, including 152 immunoglobulin like, 132 fibronectine 3 (Fn3), 19 Kelch, 14 tetratricopeptide repeat, and 15 solenoid domains [37]. The variant Arg23483His is located in the 125th of the 132 Fn3 domains (PF00041), while Gln24857His is located inside titin's only serine kinase domain (PF00069), a structurally conserved protein domain that plays an important role in the regulation of cell proliferation, apoptosis and cell differentiation. The location of this variant and other functional residues in the protein structure of the kinase domain is visualized in Fig. 3. The mutated residue Gln24857His is located opposite of the active site and results in a charge and polarity change. The variant was further predicted to destabilize the protein structure by MAESTROweb $(\Delta \Delta \mathrm{G}=1.433$, confidence $=0.8)$. In addition, the wild type residue glutamine was highly conserved in a multiple sequence alignment of homologous sequences from 63 species computed by ConSurf.
In the next analysis step, we identified 311 genes related to $P K P 2$ following the criteria defined in the Methods section: (i) Five genes form a complex with $P K P 2$, (ii) 33 genes interact with $P K P 2$, (iii) four genes are transcription factors of $P K P 2$, (iv) 275 genes are involved in one of 12 biological processes together with $P K P 2$, (v) and three genes are paralogs of PKP2. A list of these PKP2-related genes is available as Additional file 1: Table S2. Four Fam1 genes and ten Fam2 genes were in the set of PKP2-related genes and were prioritized based on their expression in the heart, the number and type of variants, their allele frequencies and deleteriousness prediction. These PKP2-related genes and variants of both families are summarized in Table 2. The full table with additional, detailed annotations is available as Additional file 1: Table S3.

Of the four PKP2-related genes DAG1, DAB2IP, and $C T B P 2$ in Fam1 are associated with PKP2 through the GO BP process "negative regulation of cell migration", while TCF25 is associated with PKP2 through the GO BP process "heart development". The variants in all four genes are predicted neutral by PROVEAN, and all but the one in CTBP2 are rare. The highest ranking PKP2-related gene in Fam1 is DAG1, which encodes for dystroglycan, a central component of the dystrophin-glycoprotein complex (DGC). Dystroglycan is post-translationally cleaved into $\alpha$ - and $\beta$-dystroglycan subunits [38]. $\alpha$-dystroglycan is an extracellular protein involved in the interactions between DGC and extracellular matrix components, while $\beta$-dystroglycan contains a single transmembrane domain and a C-terminal cytoplasmic tail. The DAG1 candidate substitution ENSP00000312435.2:p.Leu86Phe is located in the $\alpha$-dystroglycan $\mathrm{N}$-terminal region, where the leucine side chain is solvent exposed on the side of a Ig-like domain [39]. The position is in close proximity to Thr63, identified as a O-glycosylation [40] (see Additional file 1: Fig. S1). The variant was predicted to stabilize protein structure by MAESTROweb $(\Delta \Delta \mathrm{G}=-0.231$, confidence $=$ $0.9)$. For the other three candidate genes, no protein structure was available in $\mathrm{PDB}$, so predictions with MAESTROweb could not be computed.

The highest ranking PKP2-related gene in Fam2 is the interferon regulatory factor 1 (IRF1), which harbors a rare, deleterious-predicted missense variant in the affected male patient Fam2.II.1 and his healthy father Fam2.I.1. IRF1 inhibits cell growth in coronary artery smooth muscle cells [41]. Repression of IRF1 has lead to a higher susceptibility to the formation of neointima (scar tissue) following vessel injury in mice [41].

\section{Discussion}

In this study we investigated the genetic cause of ACM in two families using whole exome sequencing. Since all affected and some unaffected individuals were known to harbor PKP2 variants, we investigated whether a second 


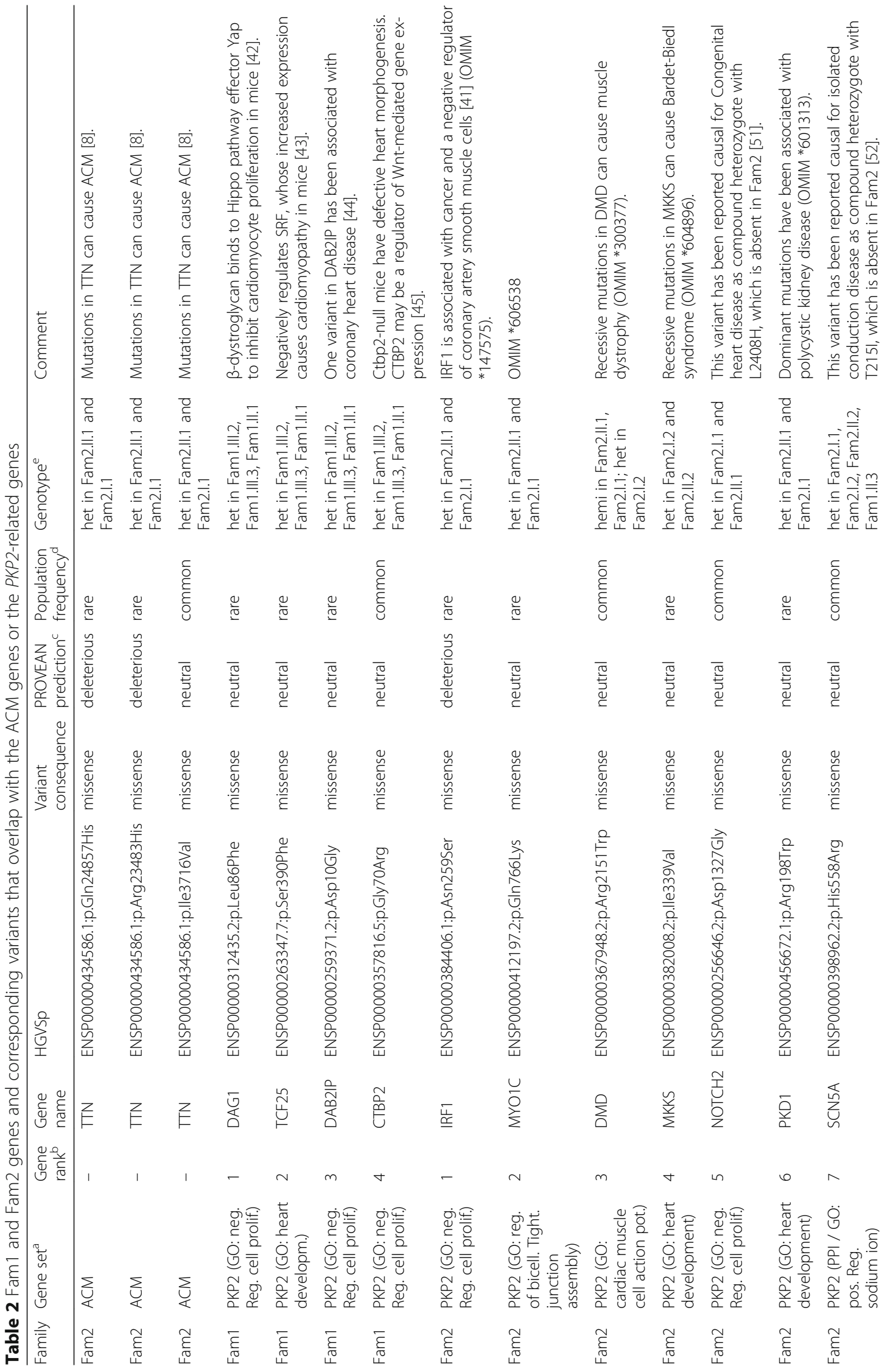




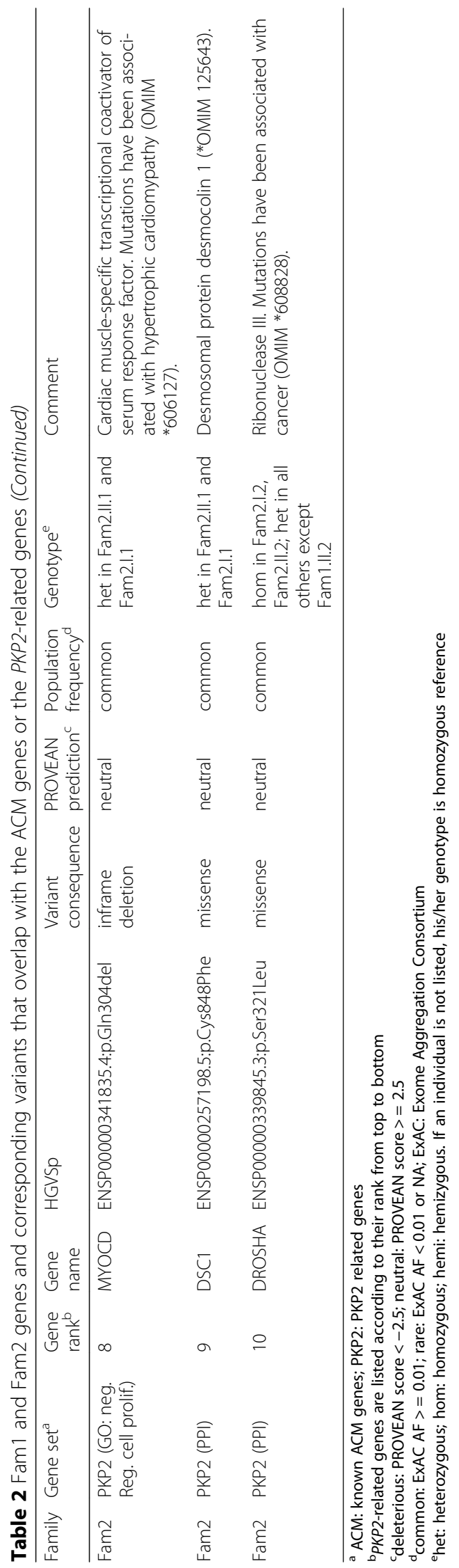




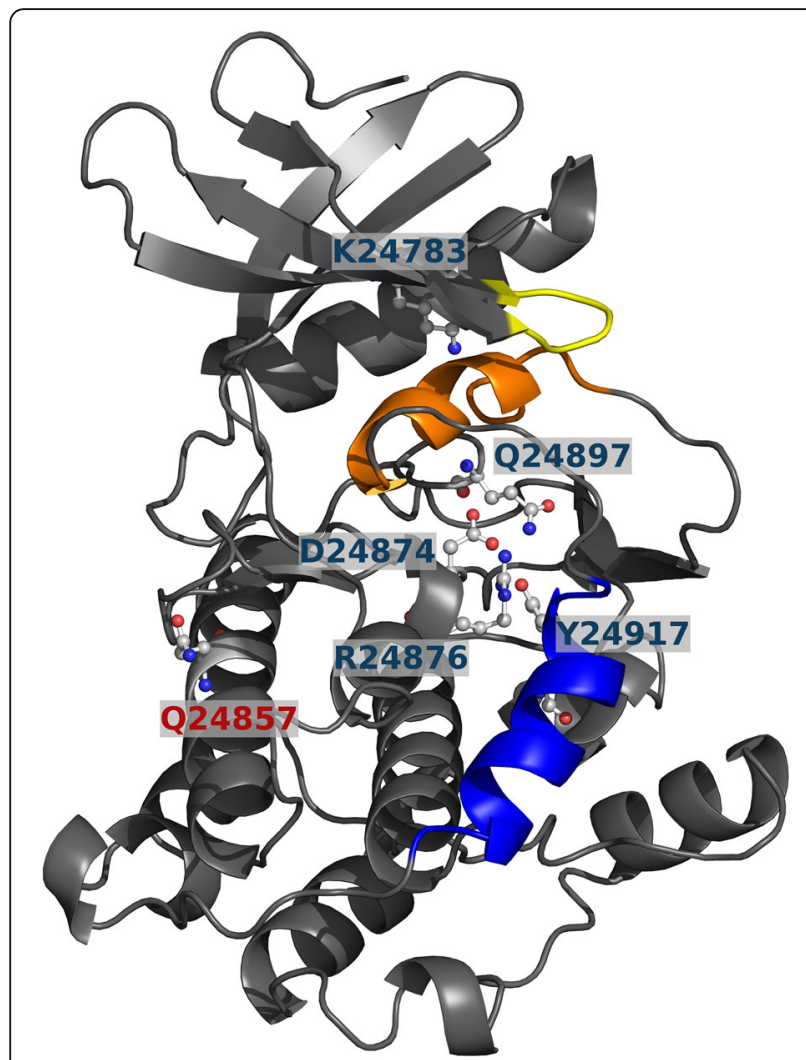

Fig. 3 Structure of the titin kinase domain (PDB 1tki chain A). Functional residues are represented as ball and stick, D24874 is the catalytic aspartate. The ATP binding site includes residue K24783 as well as the nearby yellow loop. The calcium/calmodulin binding helix is colored blue, the helix in orange blocks the ATP binding site in this autoinhibited conformation. Residue Q24857 is solvent exposed on the side opposite to the functional residues

gene was involved in a digenic inheritance pattern, with the second gene either causing ACM in the affected individuals together with $P K P 2$, or compensating the effect of the PKP2 variants in the carriers. We identified 74 and 212 genes in families 1 and 2, respectively, which carried variants consistent with the mode of digenic inheritance. To obtain results that can be easily interpreted, we restricted our analysis to genes either associated with ACM or related to $P K P 2$. In family 1 we identified four genes that are annotated with the same biological process as PKP2. In family 2 we identified the ACM associated gene TTN and ten genes related to PKP2 through a shared biological process or protein interactions (see Fig. 2).

Of the four PKP2-related Fam1 genes, the genes homologous to DAG1, TCF25, and CTBP2 have been linked to cardiomyocyte proliferation or heart development in mice and, in case of TCF25, also in human. DAG1 and TCF25 negatively regulate heart development, while a knock out of $C T B P 2$ leads to a lethal malformation of the heart in mice. A variant in DAB2IP has been associated with coronary heart disease in two studies, indicating that this gene might also play a crucial rule for the normal functioning of the heart. It has been reported that $\beta$ dystroglycan, a protein product of $D A G 1$, directly binds to the Hippo pathway effector Yap to inhibit cardiomyocyte proliferation in mice [42]. In particular, the Hippo pathway and DGC cooperatively regulate tissue growth in mouse hearts after injury. Yap and the Hippo pathway have been directly implicated in ACM pathogenesis [30]. TCF25 (previously named NULP1) was suggested as a transcription factor that negatively regulates the serum response factor (SRF). SRF controls muscle differentiation and cellular growth and regulates cardiac genes. SRF overexpression has been shown to cause cardiomyopathy and cardiac hypertrophy in mice. Therefore, TCF 25 may function as a transcriptional repressor of SRF in human heart development [43]. DAB2IP acts as a tumor suppressor gene, and is inactivated by methylation in prostate and breast cancers. A genome-wide association study found the rs7025486 variant in $D A B 2 I P$ associated with coronary heart disease, which was replicated in a second study [44]. CTBP2 encodes two proteins, a transcriptional repressor and a major component of synaptic ribbons. Silencing the homologous $C t b p 2$ gene in mice causes defects in heart morphogenesis and results in early embryonic lethality [45]. Ctbp2-null mice show similar axial truncation phenotypes as mice with mutations in some Wnt target genes, suggesting that CTBP2 may be a regulator of Wntmediated gene expression [45]. Indeed, CtBP2 acts as corepressor of $\mathrm{C} / \mathrm{EBP} \alpha$, an early regulator of adipogenesis, and target of the Wnt signaling pathway [46]. Furthermore, Sox 6 has been found to bind Ctbp 2 to repress the fibroblast growth factor 3 [47] and Sox6 to regulate the cardiac myocyte development in mice [48]. Although none of the variants in these four genes are predicted to be deleterious and the variant in DAG1 is even predicted to stabilize protein structure, they could nevertheless affect protein stability, flexibility, and interaction with the other binding partners. However, in the present study we could not find any indication that these genes may act together with PKP2 to cause the ACM phenotype in the affected individuals of Fam1.

Since TTN is a known ACM associated gene, it is a likely candidate in the second family. TTN encodes for titin, the largest human protein with isoforms ranging from about 27.000 to 36.000 amino acids. Titin is functionally linked to the desmosome (and thereby to PKP2), since titin filaments are a key component of sarcomeres and connect to the transitional junction at the intercalated disk [8]. In a cohort of 38 ACM families, Taylor et al. identified novel TTN mutations in $18 \%$ of the families [8]. In addition to ACM, TTN has been associated with dilated, hypertrophic, and restrictive cardiomyopathy [49]; its association with hypertropic cardiomyopathy, however, is still under debate [50]. The affected patient 
Fam2.II.1 and his father both harbor two rare heterozygous missense variants that are predicted deleterious. The Gln24857His variant is located in titin's only kinase domain at a conserved position and is predicted to destabilize protein structure, while Arg23483His is located in one of the $132 \mathrm{Fn} 3$ domains. Therefore, the Gln24857His variant is more likely to impair titin function than the Arg23483His variant, even though disease causal variants in repetitive titin domains have been reported [8]. Studies with transfected cell lines have shown that heterozgyous mutations, in contrast to homozygous mutations, still allow for functional sarcomeres but may alter the organizational characteristics and impair the normal cardiac function [49]. These findings agree well with the hypothesis that either one or both of these variants alter the structure of titin and only lead to ACM in combination with the PKP2 mutation.

In addition to the genes described here in more detail, there are other promising candidates for the second causal gene in Fam2 (see Table 2). For example, of the PKP2-related genes, NOTCH2 and SCN5A harbor one of two compound heterozygous variants that have been reported to cause congenital heart disease and isolated conduction disease, respectively [51, 52]; $D M D$ harbors a neutral hemizygous variant in the affected Fam2 individual, a gene where recessive variants can cause muscle dystrophy; DAG1 and $M K K S$ are associated with recessive diseases, yet the variants in the affected individuals are heterozygous; IRF1 is associated with non-cardiac diseases; $D S C 1$, a desmosomal gene not associated with heart disease, harbors a common missense variant that is predicted neutral. Since all of these genes are interesting candidates for follow up studies, it would be interesting to test whether the same or other rare variants in our candidate genes can be identified in a large cohort ACM patients, both in patients carrying desmosomal mutations or other ACM related mutations as well as in genetically unsolved cases.

Digenic inheritance has previously been reported as a disease-causal mechanism for ACM, however, these studies have focused on desmosomal genes. As a result, there are no reports of digenic inheritance in ACM with PKP2 and a non-desmosomal gene such as TTN.

We are aware of limitations in our study. Both families have relatively few members, which resulted in a large set of variants and genes as possible candidates for digenic inheritance. Only $85 \%$ of the exome was covered with at least $10 \mathrm{X}$. Since we required a minimum coverage of $10 \mathrm{X}$ to accept a variant call, $15 \%$ of the exome could not be investigated. However, coverage at the ACM genes was well above average, so it is unlikely that variants were missed in these genes. Due to the large number of candidate genes in each family, we restricted our analysis to ACM or PKP2-related genes, potentially removing causative digenic genes with unknown associations. Even though the TTN variants in
Fam 2 were confirmed by Sanger sequencing, no functional validation of the variant effects was performed. Therefore, it still needs to be shown if TTN or any of the candidate genes in Fam 1 truly cause ACM together with PKP2 in the respective family. A functional validation could be performed based on induced pluripotent stem cell (iPSC) models from the ACM-affected individuals, where the PKP2 variant or the TTN/Fam1 variants are reversed [53]. In the iPSC derived cardiomyocytes the effect of the genetic variants could be investigated by comparing fat accumulation and cell electrophysiology to the double mutant cells. Other cell models that could be employed for validation are progenitor cells (they differentiate easily in vitro), noncontractile cardiac mesenchymal stromal cells (ideal for studying lipid metabolism), or primary or immortalized cardiomyocytes (enable investigation of gap-junctions and ionchannels) [54]. Yet, even if successful, such experiments would demonstrate the mode of effect in the respective family, while general conclusions about the role of TTN/ Fam1 genes in ACM could not necessarily be drawn from them. To evaluate the roles of these genes in ACM more generally, other ACM patients carrying desmosomal variants could be checked for rare variants in the respective genes. Unfortunately, we currently do not have additional ACM patients for testing and the NCBI Sequence Read Archive does not contain public whole exome or whole genome sequence data of ACM patients. The search for genes with a digenic effect is a considerable challenge since variants in both relevant genes do not necessarily have a pathogenic effect when occurring individually [11]. The functional or structural change caused by the variant in either protein may be subtle, and may for example lead to a change at a protein binding affinity or a change in gene expression. Therefore, standard criteria usually applied to evaluate the likelihood of variant pathogenicity like rarity and computational predictions might not be well suited. Consequently, we did not exclude variants based on these criteria, yet in the absence of functional validation and more appropriate models, we prioritized and discussed our results according to these methods. We would like to recall that we did not distinguish between variants that were present in the affected and not in the carriers and variants present in the carriers but not in the affected, since we were interested in genes whose function might differ between affected individuals and carriers due to the variants. However, we point out that of the 17 variants listed in Table 2, only three (Ile339Val in MKKS, His558Arg in SCN5A, and Ser321Leu in DROSHA) are present in the carriers and not in the affected individuals, suggesting that our strategy of prioritizing based on rarity and predicted pathogenicity is appropriate. Finally, we acknowledge the possibility that more than two genes could be involved in the pathogenesis (oligogenic inheritance) or, contrarily, that environmental factors could influence the penetrance of the $P K P 2$ variants 
without other genetic variants having an effect on the development of ACM. However, in our study, the main nongenetic disease modulators (age, sex, and physical exercise) are not sufficient to explain the different phenotypic expression in affected individuals and carriers in the two analyzed families (see Table 1). In Fam1, both the carrier Fam1.III.1 and the affected Fam1.III.2 are male and are close in age, and carrier Fam1.III.1 and the affected Fam1.III.3 are both physically active. In Fam2, both the affected Fam2.II.1 and the carrier Fam2.II.2 are male, physically active, and relatively close in age.

\section{Conclusions}

In the present work we have provided further indication that a single (desmosomal) mutation might not be sufficient to cause ACM, by showing the co-segregation of other variants with PKP2 and the phenotype in two families.

\section{Additional file}

Additional file 1: Includes Supplementary Methods; Tables S1, S2, and S3; Fig. S1. (DOCX 2344 kb)

\section{Abbreviations}

ACM: Arrhythmogenic cardiomyopathy; ARVC: arrhythmogenic right ventricular cardiomyopathy; BP: biological process; CNV: copy number variation; Fam1: family 1; Fam2: family 2; Fn3: fibronectine 3; GO: Gene Ontology; iPSC: induced pluripotent stem cell; SNP: single nucleotide polymorphism

\section{Acknowledgements}

We express our gratitude to the patients and their families for their participation in this study. We thank Dr. Veronica De Sanctis and Dr. Roberto Bertorelli (NGS Facility, CIBIO-LaBSSAH, University of Trento) for NGS sequencing and helpful discussions. We acknowledge the contributions of Deborah Mascalzoni as an ethics consultant.

\section{Competing interests}

The authors declare that they have no competing interests.

\section{Funding}

The research was funded by the Department of Innovation, Research, Development and Cooperatives of the Autonomous Province of BolzanoSouth Tyrol. The funder had no influence on the design of the study, nor on collection, analysis, or interpretation of data.

\section{Availability of data and materials}

The datasets generated and/or analyzed during the study are not publicly available since the General Authorization No. 8/2014 for the Processing of Genetic Data (Italian Official Gazette, n. 301, 30 December 2014), prohibits "communication" of genetic data to third parties for not clearly defined purposes. The data are available from the corresponding author after access approval by a multidisciplinary access committee and signing of a data transfer agreement contract that defines limits, obligations, and purpose of the data usage.

\section{Authors' contributions}

EK performed the computational processing and analysis of the sequencing data and was a major contributor in writing the manuscript. CV performed the Sanger sequencing validation and added the respective methods section to the document. BMM prepared the libraries for whole exome sequencing, added the respective methods section in the manuscript, and contributed in writing the manuscript. $\mathrm{HB}$ contributed in the conception of the computational analysis and to the writing of the manuscript. AP prepared the libraries for whole exome sequencing and added the respective methods section in the document. PP critically revised the manuscript for important intellectual content. MC collected and analyzed the clinical data and critically revised the manuscript. WR collected and analyzed the clinical data and critically revised the manuscript. GP critically revised the manuscript for important intellectual content and recruited the participants for the study. VM prepared the libraries for whole exome sequencing, added the respective methods section in the manuscript, and contributed in writing the manuscript. FSD substantially contributed to the conception of the computational analysis and to the writing of the manuscript. ES recruited the participants for the study, conceived the study, and contributed to the writing of the manuscript. AR initialized and conceived the study, and contributed to the writing of the manuscript. All authors read and approved the final manuscript.

\section{Consent for publication}

Written informed consent for publication was obtained from all participants of this study.

\section{Ethics approval and consent to participate}

The study complies with the declaration of Helsinki and was approved by the ethics committee of the Centro Cardiologico Monzino-IRCCS. Written informed consent for participation was obtained from all participants.

\section{Publisher's Note}

Springer Nature remains neutral with regard to jurisdictional claims in published maps and institutional affiliations.

\section{Author details}

${ }^{1}$ Institute for Biomedicine, Eurac Research, Affiliated Institute of the University of Lübeck, Bolzano, Italy. ${ }^{2}$ Heart Rhythm Center, Centro Cardiologico Monzino IRCCS, Via Parea 4, 20138 Milan, Italy. ${ }^{3}$ General Hospital Bolzano/ Bozen, Bolzano, Italy. ${ }^{4}$ Vascular Biology and Regenerative Medicine Unit, Centro Cardiologico Monzino IRCCS, Via Parea 4, 20138 Milan, Italy.

Received: 2 March 2017 Accepted: 23 November 2017

Published online: 08 December 2017

\section{References}

1. Ohno S. The genetic background of arrhythmogenic right ventricular cardiomyopathy. J Arrhythmia. 2016;32:398-403.

2. Garcia-Gras E, Lombardi R, Giocondo MJ, Willerson JT, Schneider MD, Khoury DS, et al. Suppression of canonical Wnt/ $\beta$-catenin signaling by nuclear plakoglobin recapitulates phenotype of arrhythmogenic right ventricular cardiomyopathy. J Clin Invest. 2006;116:2012-21.

3. Chen SN, Gurha P, Lombardi R, Ruggiero A, Willerson JT, Marian AJ. The hippo pathway is activated and is a causal mechanism for adipogenesis in arrhythmogenic cardiomyopathy. Circ Res. 2014;114:454-68.

4. Ortiz-Genga MF, Cuenca S, Dal Ferro M, Zorio E, Salgado-Aranda R, Climent $V$, Padrón-Barthe $L$, et al. Truncating FLNC mutations are associated with high-risk dilated and Arrhythmogenic cardiomyopathies. J Am Coll Cardiol. 2016;68:2440-51.

5. Mayosi BM, Fish M, Shaboodien G, Mastantuono E, Kraus S, Wieland T, et al. Identification of cadherin $2(\mathrm{CDH} 2)$ mutations in Arrhythmogenic right ventricular cardiomyopathy. Circ Cardiovasc Genet. 2017;10(2)

6. Sen-Chowdhry S, Syrris P, Pantazis A, Quarta G, McKenna WJ, Chambers JC. Mutational heterogeneity, modifier genes, and environmental influences contribute to phenotypic diversity of arrhythmogenic cardiomyopathy. Circ Cardiovasc Genet. 2010;3:323-30.

7. Severini GM, Krajinovic M, Pinamonti B, Sinagra G, Fioretti P, Brunazzi MC, Falaschi A, Camerini F, Giacca M, Mestroni LA. New locus for Arrhythmogenic right ventricular dysplasia on the long arm of chromosome 14. Genomics. 1996;31:193-200.

8. Taylor M, Graw S, Sinagra G, Barnes C, Slavov D, Brun F, et al. Genetic variation in titin in arrhythmogenic right ventricular cardiomyopathy-overlap syndromes. Circulation. 2011;124:876-85.

9. Li D, Ahmad F, Gardner MJ, Weilbaecher D, Hill R, Karibe A, et al. The locus of a novel gene responsible for Arrhythmogenic right-ventricular dysplasia characterized by early onset and high penetrance maps to chromosome 10p12-p14. Am J Hum Genet. 2000;66:148-56. 
10. Andreasen C, Nielsen JB, Refsgaard L, Holst AG, Christensen AH, Andreasen $L$, et al. New population-based exome data are questioning the pathogenicity of previously cardiomyopathy-associated genetic variants. Eur J Hum Genet. 2013;21:918-28.

11. Cooper DN, Krawczak M, Polychronakos C, Tyler-Smith C, Kehrer-Sawatzki H. Where genotype is not predictive of phenotype: towards an understanding of the molecular basis of reduced penetrance in human inherited disease. Hum Genet. 2013;132:1077-130.

12. Xu T, Yang Z, Vatta M, Rampazzo A, Beffagna G, Pillichou K, et al. Compound and Digenic heterozygosity contributes to Arrhythmogenic right ventricular cardiomyopathy. J Am Coll Cardiol. 2010;55:587-97.

13. Rasmussen TB, Palmfeldt J, Nissen PH, Magnoni R, Dalager S, Jensen UB, et al. Mutated Desmoglein-2 proteins are incorporated into desmosomes and exhibit dominant-negative effects in Arrhythmogenic right ventricular cardiomyopathy. Hum Mutat. 2013;34:697-705.

14. Nakajima T, Kaneko Y, Irie T, Takahashi R, Kato T, lijima T, Iso T, Kurabayashi M. Compound and digenic heterozygosity in desmosome genes as a cause of arrhythmogenic right ventricular cardiomyopathy in Japanese patients. Circ J. 2012;76:737-43.

15. Génin E, Feingold J, Clerget-Darpoux F. Identifying modifier genes of monogenic disease: strategies and difficulties. Hum Genet. 2008;124:357-68.

16. Meyer $S$, van der Meer $P$, van Tintelen JP, van den Berg MP. Sex differences in cardiomyopathies. Eur J Heart Fail. 2014;16:238-47.

17. James CA, Bhonsale A, Tichnell C, Murray B, Russell SD, Tandri H, et al. Exercise increases age-related penetrance and arrhythmic risk in arrhythmogenic right ventricular dysplasia/cardiomyopathy-associated desmosomal mutation carriers. J Am Coll Cardiol. 2013;62:1290-2097.

18. Marcus Fl, McKenna WJ, Sherrill D, Basso C, Bauce B, Bluemke DA, et al. Diagnosis of arrhythmogenic right ventricular cardiomyopathy/dysplasia. Eur Heart J. 2010:31:806-14.

19. Li H, Durbin R. Fast and accurate short read alignment with burrowswheeler transform. Bioinformatics. 2009;25:1754-60.

20. McKenna A, Hanna M, Banks E, Sivachenko A, Cibulskis K, Kernytsky A, et al. The genome analysis toolkit: a MapReduce framework for analyzing nextgeneration DNA sequencing data. Genome Res. 2010;20:1297-303.

21. Kersey PJ, Allen JE, Armean I, Boddu S, Bolt BJ, Carvalho-Silva D, et al. Ensembl genomes 2016: more genomes, more complexity. Nucleic Acids Res. 2016:44:D574-80.

22. Lek M, Karczewski KJ, Samocha KE, Banks E, Fennell T, AH O, et al. Analysis of protein-coding genetic variation in 60,706 humans. Nature. 2016:285-91.

23. Choi Y, Sims GE, Murphy S, Miller JR, Chan AP. Predicting the functional effect of amino acid substitutions and Indels. PLoS One. 2012;7:e46688.

24. König E, Rainer J, Domingues FS. Computational assessment of feature combinations for pathogenic variant prediction. Mol Genet Genomic Med. 2016:4:431-46

25. Fromer M, Purcell SM. Using XHMM software to detect copy number variation in whole-exome sequencing data. Curr Protoc Hum Genet. 2014; 91:597-607.

26. Laimer J, Hofer H, Fritz M, Wegenkittl S, Lackner P. MAESTRO-multi agent stability prediction upon point mutations. BMC Bioinformatics. 2015;16:116.

27. Laimer J, Hiebl-Flach J, Lengauer D, Lackner P. MAESTROweb: a web server for structure-based protein stability prediction. Bioinformatics. 2016;32:1414-6.

28. Glaser F, Pupko T, Paz I, Bell RE, Bechor-Shental D, Martz E, et al. ConSurf: identification of functional regions in proteins by surface-mapping of phylogenetic information. Bioinformatics. 2003;19:163-4.

29. Uhlén M, Fagerberg L, Hallström BM, Lindskog C, Oksvold P, Mardinoglu A, et al. Tissue-based map of the human proteome. Science. 2015;347:1260419.

30. Rampazzo A, Calore M, Van Hengel J, Van Roy F. Intercalated discs and arrhythmogenic cardiomyopathy. Circ Cardiovasc Genet. 2014;7:930-40.

31. Huttlin EL, Ting L, Bruckner RJ, Gebreab F, Gygi MP, Szpyt J, et al. The BioPlex network: a systematic exploration of the human interactome. Cell. 2015;162:425-40.

32. Szklarczyk D, Franceschini A, Wyder S, Forslund K, Heller D, Huerta-Cepas J, et al. STRING v10: protein-protein interaction networks, integrated over the tree of life. Nucleic Acids Res. 2014;43:D447-52.

33. Calderone A, Castagnoli L, Cesareni G. Mentha: a resource for browsing integrated protein-interaction networks. Nat Meth. 2013;10:690-1.

34. Lesurf R, Cotto KC, Wang G, Griffith M, Kasaian K, Jones SJM, et al. The open regulatory annotation consortium. ORegAnno 3.0: a communitydriven resource for curated regulatory annotation. Nucleic Acids Res. 2016;44:D126-32.
35. Gene Ontology Consortium. Gene ontology consortium: going forward. Nucleic Acids Res. 2015:43:D1049-56.

36. Weichenberger CX, Blankenburg H, Palermo A, D'Elia Y, König E, Bernstein E, et al. Dintor: functional annotation of genomic and proteomic data. BMC Genomics. 2015;16:1081.

37. Chauveau C, Rowell J, Ferreiro AA. Rising titan: TTN review and mutation update. Hum Mutat. 2014;35:1046-59.

38. Barresi R. Dystroglycan: from biosynthesis to pathogenesis of human disease. J Cell Sci. 2006;119:199-207.

39. Covaceuszach S, Bozzi M, Bigotti MG, Sciandra F, Konarev PV, Brancaccio A, et al. Structural flexibility of human a-dystroglycan. FEBS Open Bio. 2017;7:1064-77.

40. Halim A, Rüetschi U, Larson G, Nilsson J. LC-MS/MS characterization of Oglycosylation sites and glycan structures of human cerebrospinal fluid glycoproteins. J Proteome Res. 2013;12:573-84.

41. Wessely $R$, Hengst $L$, Jaschke B, Wegener F, Richter T, Lupetti R, et al. A central role of interferon regulatory factor- 1 for the limitation of neointimal hyperplasia. Hum Mol Genet. 2003;12:177-87.

42. Morikawa Y, Heallen T, Leach J, Xiao Y, Martin JF. Dystrophin-glycoprotein complex sequesters yap to inhibit cardiomyocyte proliferation. Nature. 2017; 547:227-31.

43. Cai Z, Wang Y, Yu W, Xiao J, Li Y, Liu L, et al. Hnulp1, a basic helix-loop-helix protein with a novel transcriptional repressive domain, inhibits transcriptional activity of serum response factor. Biochem Biophys Res Commun. 2006;343:973-81.

44. Harrison SC, Cooper JA, Li K, Talmud PJ, Sofat R, Stephens JW, et al. Association of a sequence variant in DAB2IP with coronary heart disease. Eur Heart J. 2012;33:881-8.

45. Chinnadurai G. CtBP family proteins: more than transcriptional corepressors. BioEssays. 2003;25:9-12.

46. Vernochet C, Peres SB, Davis KE, McDonald ME, Qiang L, Wang H, et al. C/ $\mathrm{EBP}$ and the corepressors $\mathrm{CtBP} 1$ and $\mathrm{CtBP} 2$ regulate repression of select visceral white adipose genes during induction of the Brown phenotype in white adipocytes by peroxisome proliferator-activated receptor agonists. Mol Cell Biol. 2009:29:4714-28.

47. Murakami A, Ishida S, Thurlow J, Revest JM, Dickson C. SOX6 binds CtBP2 to repress transcription from the Fgf-3 promoter. Nucleic Acids Res. 2001;29: 3347-55.

48. Cohen-Barak O, Yi Z, Hagiwara N, Monzen K, Komuro I, Brilliant MH. Sox6 regulation of cardiac myocyte development. Nucleic Acids Res. 2003;31: 5941-8.

49. Neiva-Sousa M, Almeida-Coelho J, Falcão-Pires I, Leite-Moreira AF. Titin mutations: the fall of goliath. Heart Fail Rev. 2015;20:579-88.

50. Herman DS, Lam L, Taylor MRG, Wang L, Teekakirikul P, Christodoulou D, et al. Truncations of titin causing dilated cardiomyopathy. N Engl J Med. 2012; 366:619-28.

51. Priest JR, Osoegawa K, Mohammed N, Nanda V, Kundu R, Schultz K, et al. De novo and rare variants at multiple loci support the Oligogenic origins of atrioventricular septal heart defects. PLoS Genet. 2016;12:e1005963.

52. Viswanathan PC, Benson DW, Balser JRA. Common SCN5A polymorphism modulates the biophysical effects of an SCN5A mutation. J Clin Invest. 2003 111:341-6.

53. Hockemeyer D, Jaenisch R. Induced pluripotent stem cells meet genome editing. Cell Stem Cell. 2016;18:573-86.

54. Sommariva E, Stadiotti I, Perrucci GL, Tondo C, Pompilio G. Cell models of arrhythmogenic cardiomyopathy: advances and opportunities. Dis Model Mech. 2017:10:823-35. 